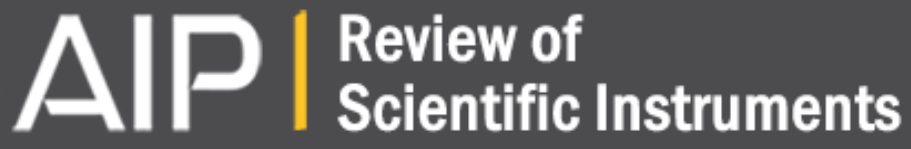

Generation of circularly polarized radiation from a compact plasma-based extreme ultraviolet light source for tabletop X-ray magnetic circular dichroism studies

Daniel Wilson, Denis Rudolf, Christian Weier, Roman Adam, Gerrit Winkler, Robert Frömter, Serhiy Danylyuk, Klaus Bergmann, Detlev Grützmacher, Claus M. Schneider, and Larissa Juschkin

Citation: Review of Scientific Instruments 85, 103110 (2014); doi: 10.1063/1.4897491

View online: http://dx.doi.org/10.1063/1.4897491

View Table of Contents: http://scitation.aip.org/content/aip/journal/rsi/85/10?ver=pdfcov

Published by the AIP Publishing

\section{Articles you may be interested in}

Room temperature magnetic stabilization of buried cobalt nanoclusters within a ferromagnetic matrix studied by soft x-ray magnetic circular dichroism

Appl. Phys. Lett. 93, 172511 (2008); 10.1063/1.3012368

Mn L3,2 Xray Absorption Spectroscopy And Magnetic Circular Dichroism In Ferromagnetic Ga1-x Mn x P AIP Conf. Proc. 893, 1177 (2007); 10.1063/1.2730317

Facility for combined in situ magnetron sputtering and soft $\mathrm{x}$-ray magnetic circular dichroism

Rev. Sci. Instrum. 77, 073903 (2006); 10.1063/1.2219719

Photovoltage detection of $\mathrm{x}$-ray absorption and magnetic circular dichroism spectra of magnetic films grown on semiconductors

J. Appl. Phys. 93, 2028 (2003); 10.1063/1.1537453

A direct two-dimensional comparison of magnetic circular dichroism and magnetic linear dichroism in ultraviolet photoemission spectroscopy

J. Appl. Phys. 91, 7364 (2002); 10.1063/1.1456425

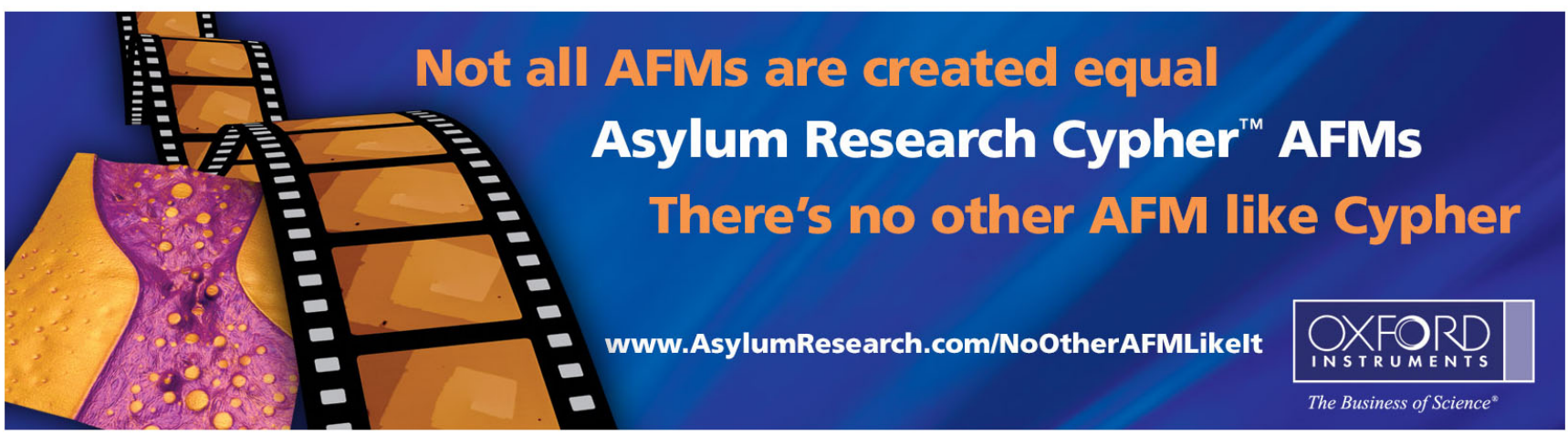




\title{
Generation of circularly polarized radiation from a compact plasma-based extreme ultraviolet light source for tabletop X-ray magnetic circular dichroism studies
}

\author{
Daniel Wilson, ${ }^{1,2, a)}$ Denis Rudolf, ${ }^{1,2, a), b)}$ Christian Weier, ${ }^{3}$ Roman Adam, ${ }^{3}$ Gerrit Winkler, ${ }^{4}$ \\ Robert Frömter, ${ }^{4}$ Serhiy Danylyuk, ${ }^{5}$ Klaus Bergmann, ${ }^{6}$ Detlev Grützmacher, ${ }^{2}$ \\ Claus M. Schneider, ${ }^{3}$ and Larissa Juschkin ${ }^{1,2}$ \\ ${ }^{1}$ RWTH Aachen University, Experimental Physics of EUV, Steinbachstraße 15, 52074 Aachen, Germany \\ ${ }^{2}$ Forschungszentrum Jülich GmbH, Peter Grünberg Institut (PGI-9), JARA-FIT, 52425 Jülich, Germany \\ ${ }^{3}$ Forschungszentrum Jülich GmbH, Peter Grünberg Institut (PGI-6), JARA-FIT, 52425 Jülich, Germany \\ ${ }^{4}$ Institut für Angewandte Physik, Universität Hamburg, Jungiusstraße 11, 20355 Hamburg, Germany \\ ${ }^{5}$ RWTH Aachen University, Chair for Technology of Optical Systems, JARA-FIT, Steinbachstraße 15, 52074 \\ Aachen, Germany \\ ${ }^{6}$ Fraunhofer Institute for Laser Technology, Steinbachstrasse 15, 52074 Aachen, Germany
}

(Received 1 September 2014; accepted 27 September 2014; published online 16 October 2014)

\begin{abstract}
Generation of circularly polarized light in the extreme ultraviolet (EUV) spectral region (about $25 \mathrm{eV}-250 \mathrm{eV}$ ) is highly desirable for applications in spectroscopy and microscopy but very challenging to achieve in a small-scale laboratory. We present a compact apparatus for generation of linearly and circularly polarized EUV radiation from a gas-discharge plasma light source between $50 \mathrm{eV}$ and $70 \mathrm{eV}$ photon energy. In this spectral range, the $3 p$ absorption edges of $\mathrm{Fe}(54 \mathrm{eV}), \mathrm{Co}$ $(60 \mathrm{eV})$, and $\mathrm{Ni}(67 \mathrm{eV})$ offer a high magnetic contrast often employed for magneto-optical and electron spectroscopy as well as for magnetic imaging. We simulated and designed an instrument for generation of linearly and circularly polarized EUV radiation and performed polarimetric measurements of the degree of linear and circular polarization. Furthermore, we demonstrate first measurements of the X-ray magnetic circular dichroism at the Co $3 p$ absorption edge with a plasma-based EUV light source. Our approach opens the door for laboratory-based, element-selective spectroscopy of magnetic materials and spectro-microscopy of ferromagnetic domains. (C) 2014 AIP Publishing LLC. [http://dx.doi.org/10.1063/1.4897491]
\end{abstract}

\section{INTRODUCTION}

Extreme ultraviolet (EUV) and soft X-ray spectral region extends from about $25 \mathrm{eV}$ to $12000 \mathrm{eV}$ and is dominated by strong light-matter interaction. ${ }^{1}$ The presence of absorption edges of every element allows strong elemental and chemical selectivity. In particular, linearly and circularly polarized EUV and soft X-ray radiation is highly desired for applications, such as reflectometry, ellipsometry, lithography, magneto-optical spectroscopy, and photoemission studies. In reflectometry, linearly polarized light is used for the characterization of EUV optics, such as multilayer Bragg mirrors. ${ }^{2}$ In interference lithography, the contrast between the highest and lowest intensity in the resist is considerably better with linearly polarized light compared to unpolarized light. ${ }^{3}$ Another application of polarized EUV and soft X-ray radiation, magneto-optical polarization spectroscopy, ${ }^{4-6}$ provides valuable information about magneto-optical constants and enables studies of element- and layer-selective magnetization. For magneto-optical spectroscopy, both linearly and circularly polarized light is required.

In particular, X-ray magnetic circular dichroism (XMCD) is frequently used for magneto-optical and

\footnotetext{
a) D. Wilson and D. Rudolf contributed equally to this work.

b) Author to whom correspondence should be addressed. Electronic mail: d.rudolf@fz-juelich.de
}

photoemission spectroscopy at the $2 p(700 \mathrm{eV}-860 \mathrm{eV})$ and $3 p(50 \mathrm{eV}-70 \mathrm{eV})$ absorption edges of $\mathrm{Fe}, \mathrm{Co}$, and $\mathrm{Ni}^{4-8}$

Linearly and circularly polarized EUV and soft X-ray radiation is routinely generated at large-scale facilities such as electron storage rings and free-electron lasers - unique sources of high energy photons in terms of intensity, photon energy range, spectral bandwidth, pulse duration, and polarization. Therefore, a complete polarization analysis is usually performed at synchrotrons with sophisticated polarimetric and ellipsometric instruments. ${ }^{9-11}$

In a small-scale laboratory, various EUV and soft X-ray light sources are available, but only few of them are a priori polarized. While linearly polarized EUV light is routinely generated by intense ultrashort laser pulses, ${ }^{12}$ only few attempts to either polarize EUV radiation circularly ${ }^{13,14}$ or directly generate circular EUV radiation from a femtosecond laser ${ }^{15}$ have been reported from laboratory-based experiments. A straightforward concept for conversion of linear to circular EUV polarization is to exploit the phase shift between the s- and p-components of light upon reflection from a flat surface. For that purpose, a phase shift of $\pm 90^{\circ}$ between the $\mathrm{s}$ - and p-components and identical reflectivity for the s- and pcomponents are required. ${ }^{13,14,16,17}$ Laboratory-based instruments for generation of circularly polarized EUV light employ up to four mirrors. Due to the low overall reflectivity of a few percent in the EUV spectral range a sufficiently intense 
EUV light source is required to obtain a reasonable photon flux after the conversion.

For our studies, we employ an intense gas-discharge plasma-based EUV light source. ${ }^{18-20}$ The multiply ionized atoms, in our case oxygen and nitrogen ions, emit narrowbandwidth spectral lines $\left(\Delta E / E=10^{-3}-10^{-5}\right.$, where $E$ is the photon energy) in the photon energy range between vacuum ultraviolet and soft X-rays. We optimized the EUV light source for operation above $50 \mathrm{eV}$ photon energy. To linearly polarize the initially unpolarized EUV light and simultaneously select emission lines around the $3 p$ absorption edge of Co $(60 \mathrm{eV})$, we designed a Bragg mirror linear polarizer operating close to the Brewster angle. Behind the linear polarizer, we placed a broadband triple-reflection circular polarizer, which covers the $3 p$ absorption edges of $\mathrm{Fe}, \mathrm{Co}$, and $\mathrm{Ni}$ between $50 \mathrm{eV}$ and $70 \mathrm{eV}$.

To our knowledge, for the first time in a laboratorybased experiment with a plasma-based EUV light source, we demonstrate XMCD measurements on Co/Pt-multilayer films at the Co $3 p$ absorption edge.

Our paper is structured as follows. In Sec. II, we present the simulation and design of the instrument for generation of circularly polarized EUV radiation covering the $3 p$ absorption edges of $\mathrm{Fe}, \mathrm{Co}$, and $\mathrm{Ni}$. In $\mathrm{Sec}$. III, we describe the spectral reflectivity of the Bragg mirror linear polarizer designed to reflect emission lines around the Co $3 p$ absorption edge. In Sec. IV, we analyze the performance of linear and circular polarizer around $60.5 \mathrm{eV}$ extracted from polarimetric measurements. Finally in Sec. V, we present X-ray magnetic circular dichroism measurements on $\mathrm{Co} / \mathrm{Pt}$-multilayers at the Co $3 p$ absorption edge.

\section{SIMULATION AND DESIGN OF THE INSTRUMENT FOR GENERATION OF CIRCULARLY POLARIZED EUV RADIATION AT THE 3p ABSORPTION EDGES OF IRON, COBALT, AND NICKEL}

\section{A. Simulation based on Stokes formalism}

The concept of experimental apparatus to generate circularly polarized light at the $3 p$ absorption edges of $\mathrm{Fe}, \mathrm{Co}$ and $\mathrm{Ni}$ and to measure the degree of circular polarization is presented in Fig. 1. We refer to the angle notation of Fig. 1 for further discussion of the simulation and experimental results.

To simulate and design the instrument for generation of circularly polarized light between $50 \mathrm{eV}$ and $70 \mathrm{eV}$, we applied the Stokes polarization formalism ${ }^{13,14,21}$ for our specific case. The four Stokes parameters are

$$
\begin{aligned}
& S_{0}=E_{p}^{2}+E_{s}^{2}, \\
& S_{1}=E_{p}^{2}-E_{s}^{2}, \\
& S_{2}=2 E_{p} E_{s} \cos (\Delta), \\
& S_{3}=-2 E_{p} E_{s} \sin (\Delta),
\end{aligned}
$$

which include the amplitudes of s- and p-polarized light $E_{s}$ and $E_{p}$ and the phase shift $\Delta$ between them describing the complete polarization state of light. ${ }^{21}$ To measure the degree of linear $\left(p_{L}\right)$ and circular $\left(p_{C}\right)$ polarization, we define

$$
p_{L}=\frac{S_{1}}{S_{0}}
$$

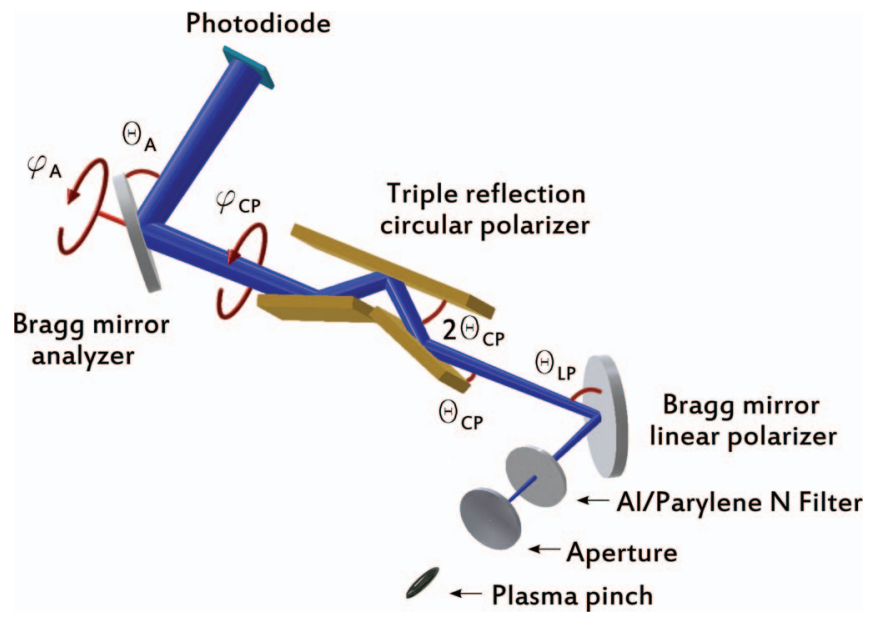

FIG. 1. The EUV light at the $3 p$ absorption edge of Co $(60 \mathrm{eV})$ emitted by the oxygen plasma first passes through an aperture and $\mathrm{Al} / \mathrm{Parylene} \mathrm{N}$ filter. Then the light is linearly (s) polarized by a Bragg mirror placed at the Brewster angle $\theta_{L P}$. The linearly polarized light is reflected by three mirrors whose rotation angle $\varphi_{C P}$ is adjusted to create circular polarization. The polarization state of light is observed by the Bragg mirror analyzer together with a photodiode, both rotatable around the beam axis $\left(\right.$ angle $\left.\varphi_{A}\right)$.

and

$$
p_{C}=\frac{S_{3}}{S_{0}} .
$$

Equation (2) assumes that the light is either completely sor p-polarized $\left(S_{2}=0\right)$. Moreover, Eqs. (2) and (3) distinguish between $\mathrm{s}\left(p_{L}=-1\right)$ - and $\mathrm{p}\left(p_{L}=+1\right)$-polarization as well as between positive $\left(p_{C}=+1\right)$ and negative $\left(p_{C}=-1\right)$ helicity (right- and left-circularly polarized light), respectively.

Each optical element, represented by a $4 \times 4$ so-called Müller matrix, has a different reflection or transmission for the s- and p-component and, in addition to that, it causes a phase shift between the two components. To characterize the polarization state of light, in our case after reflection, the two parameters $\psi_{r}$ (rotation of the main ellipse axis) and $\Delta_{r}$ (phase shift between the s- and p-component) are fundamental quantities for ellipsometric studies. ${ }^{21}$ For complex-valued s- and p-reflectivities $r_{s}$ and $r_{p}$, the ellipsometric relationship reads

$$
\tan \left(\psi_{r}\right) \times e^{i \Delta_{r}}=\frac{r_{p}}{r_{s}} .
$$

The s- and p-reflectivities, readily calculated using Fresnel equations, depend on the refractive index (and thus the photon energy) as well as the angle of incidence. For our simulations, we extracted the complex-valued refractive indices for the EUV spectral range from the database of the Center of X-Ray Optics (CXRO). ${ }^{22}$ Since the emitted radiation of our plasma discharge source is initially unpolarized, the light has to be polarized linearly first and then polarized circularly. To this end, we designed multilayer Bragg mirrors for Brewster angle operation and peak reflectivity at $53.9 \mathrm{eV}$ (Fe $3 p$ ), $60.5 \mathrm{eV}$ (Co $3 p$ ), and $67.0 \mathrm{eV}(\mathrm{Ni} 3 p)$. The requirements for the linear polarizer are high reflectivity on one hand and high degree of linear polarization on the other hand, both at the $3 p$ absorption edges of $\mathrm{Fe}, \mathrm{Co}$, and $\mathrm{Ni}$. In addition to that, a 
(a)

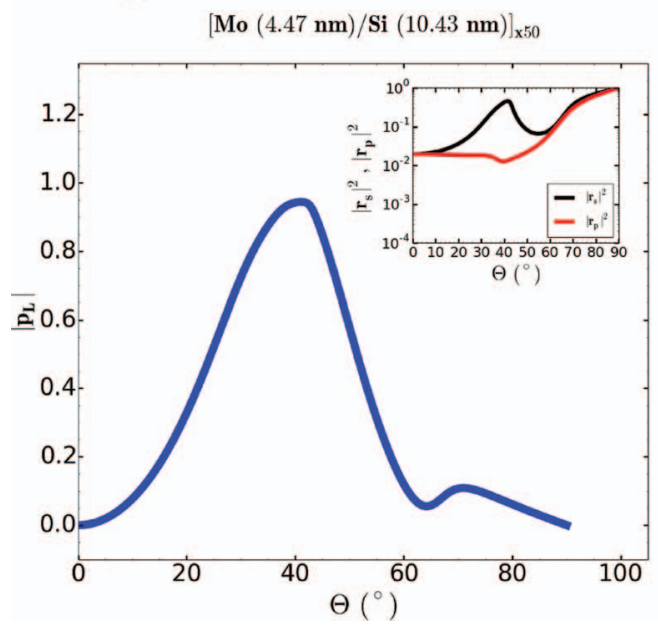

(b)

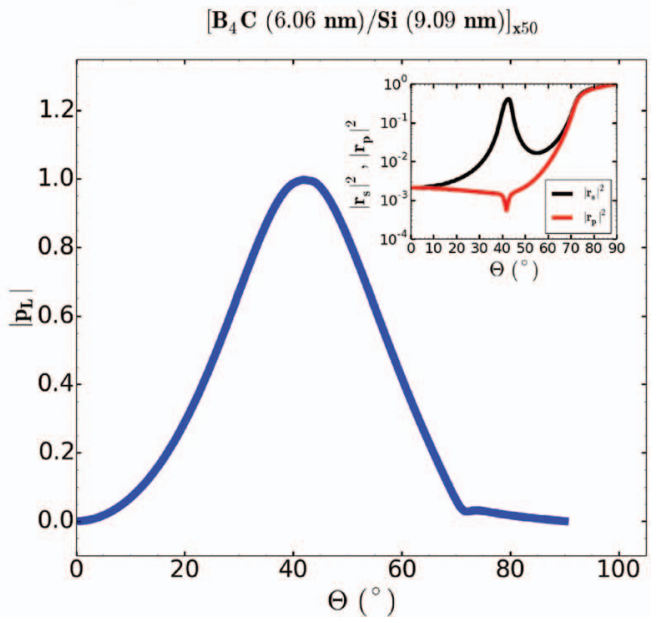

FIG. 2. The inset shows a comparison of reflectivity for s- and p-polarized light between the Mo/Si (a) and $\mathrm{B}_{4} \mathrm{C} / \mathrm{Si}$ (b) Bragg mirror linear polarizer at $60.5 \mathrm{eV}$ photon energy ( $3 p$ absorption edge of $\mathrm{Co}$ ). The simulated reflectivity for s- and p-polarized light (black and red lines) is plotted for different angles $\Theta$ with respect to normal incidence. The resulting degree of linear polarization $p_{L}$ (blue line) is higher for $\mathrm{B}_{4} \mathrm{C} / \mathrm{Si}$ linear polarizer $\left(\left|p_{L}\right|=1.00\right)$ compared to $\mathrm{Mo} / \mathrm{Si}$ linear polarizer $\left(\left|p_{L}\right|=0.94\right)$.

spectrally sufficiently narrow reflectivity peak is required to select single emission lines.

We simulated the Bragg mirror reflectivity based on the iterative algorithm from Ref. 21. In our simulations, we compared the s- and p-reflectivity of two material combinations, namely, $\mathrm{Mo} / \mathrm{Si}$ and $\mathrm{B}_{4} \mathrm{C} / \mathrm{Si}$ at the $3 p$ absorption edges of $\mathrm{Fe}$, $\mathrm{Co}$, and $\mathrm{Ni}$ (Fig. 2 for Co $3 p$ ). For the $\mathrm{B}_{4} \mathrm{C} / \mathrm{Si}$ Bragg mirror linear polarizer, our simulation shows a higher ratio between the s- and p-reflectivity in the vicinity of the Brewster angle compared to the Mo/Si linear polarizer. Due to the high degree of linear polarization $p_{L}$ close to 1 and also due to a sufficiently narrow bandwidth (1.36 nm for Co $3 p$, see Table I), we used the $\mathrm{B}_{4} \mathrm{C} / \mathrm{Si}$ Bragg mirror linear polarizer in our experiments. We summarize the most important parameters of the $\mathrm{B}_{4} \mathrm{C} / \mathrm{Si}$ Bragg mirrors in Table I. In Fig. 2, we display the simulation results for the $3 p$ absorption edge of Co $(60 \mathrm{eV})$. For the $3 p$ absorption edges of $\mathrm{Fe}$ and $\mathrm{Ni}$, the simulation results are similar in terms of magnitude and shape of the $p_{L}-\Theta$ graph.

In the next step, we simulated the degree of circular polarization due to the phase shift $\Delta$ between the s- and

TABLE I. Parameters of the $\mathrm{B}_{4} \mathrm{C} / \mathrm{Si}$ multilayer mirror linear polarizers for the $3 p$ absorption edges of $\mathrm{Fe}(53.9 \mathrm{eV})$, $\mathrm{Co}(60.5 \mathrm{eV})$, and $\mathrm{Ni}(67.0 \mathrm{eV})$. $E$ denotes the photon energy, $\theta_{B}$ the Brewster angle (with respect to normal incidence), $R_{s}$ and $R_{p}$ the s- and p-reflectivities, and $p_{L}$ the degree of linear polarization after reflection.

\begin{tabular}{lccc}
\hline \hline & {$\left[\mathrm{B}_{4} \mathrm{C}(5.36 \mathrm{~nm}) /\right.$} & {$\left[\mathrm{B}_{4} \mathrm{C}(6.06 \mathrm{~nm}) /\right.$} & {$\left[\mathrm{B}_{4} \mathrm{C}(6.72 \mathrm{~nm}) /\right.$} \\
& $\mathrm{Si}(8.04 \mathrm{~nm})]_{x 50}$ & $\mathrm{Si}(9.09 \mathrm{~nm})]_{x 50}$ & $\mathrm{Si}(10.08 \mathrm{~nm})]_{x 50}$ \\
\hline$E(\mathrm{eV})$ & 67.0 & 60.5 & 53.9 \\
$\theta_{B}(\mathrm{deg})$ & 42.79 & 42.52 & 40.81 \\
$R_{s}$ & 0.41 & 0.42 & 0.39 \\
$R_{p}$ & $0.96 \times 10^{-3}$ & $0.26 \times 10^{-3}$ & $0.72 \times 10^{-3}$ \\
$F W H M$ & 1.05 & 1.36 & 1.71 \\
of $R_{s}(\mathrm{~nm})$ & & & -0.996 \\
$p_{L}$ & -0.999 & -0.995 & \\
\hline \hline
\end{tabular}

p-components of the electric field upon reflection. Reported concepts of a circular polarizer between $50 \mathrm{eV}$ and $70 \mathrm{eV}$ photon energy ${ }^{14,16}$ are based on four non-rotatable mirrors to create a phase shift $\Delta= \pm 90^{\circ}$.

To fulfill the requirements of high degree of circular polarization $p_{C}$, high overall reflectivity of the circular polarizer between $50 \mathrm{eV}$ and $70 \mathrm{eV}$, and a simple rotation around the beam axis without any beam movement, we used three instead of four mirrors. ${ }^{23}$

For $20 \mathrm{~nm}$ Mo on $\mathrm{Si}$, we found the triple-reflection at $20^{\circ}-40^{\circ}-20^{\circ}$ grazing incidence to give the highest $\left|p_{C}\right|$ of $>0.99$ at $60.5 \mathrm{eV}$. The total phase shift after three reflections amounts to $\Delta_{C P}=-90.9^{\circ}$. The degree of circular polarization $p_{C}$ depends on the rotation angle $\varphi_{C P}$ (see Fig. 1) and amounts to $p_{C}=+1$ (right circular) for $\varphi_{C P} \approx 70^{\circ}\left(250^{\circ}\right)$ and to $p_{C}=-1$ (left circular) for $\varphi_{C P} \approx 110^{\circ}\left(290^{\circ}\right)$ for the $3 p$ absorption edges of $\mathrm{Fe}, \mathrm{Co}$, and $\mathrm{Ni}$ (Fig. 3). Therefore, the circular polarizer offers two main advantages. First, the helicity of light is readily changed between left and right circular polarization. Second, only one circular polarizer covers all $3 p$ absorption edges of the $3 d$ ferromagnets and therefore enables magneto-optical polarization spectroscopy and microscopy of $\mathrm{Fe}, \mathrm{Co}$, and Ni taking advantage of the XMCD effect. The overall reflectivity $R_{C P}$ of the circular polarizer at the $3 p$ absorption edges of $\mathrm{Fe}, \mathrm{Co}$, and $\mathrm{Ni}$ starting from fully s-polarized light is displayed in Fig. 3(b). For $p_{C}=1$ $\left(\varphi_{C P}=70^{\circ}\right)$, it amounts to about $1 \%$. Our result is comparable to the overall reflectivity obtained with the four mirror configuration. ${ }^{14,16}$

To measure the degree of linear and circular polarization, we used a Bragg mirror analyzer and a photodiode, which we rotated around the beam axis in the Rabinovitch polarimeter configuration. $^{24}$ The requirements for the Bragg mirror analyzer are the same as for the linear polarizer (high reflectivity, high degree of linear polarization, narrow bandwidth) and therefore, we used two identical Bragg mirrors for linear polarizer and analyzer. 
(a)

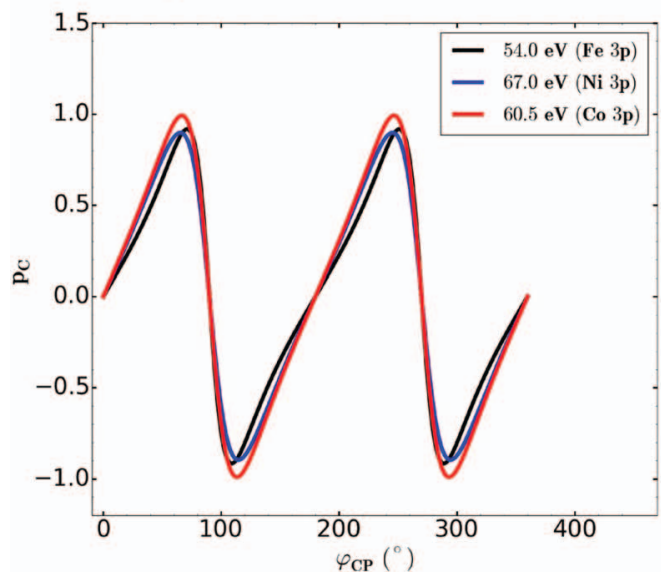

(b)

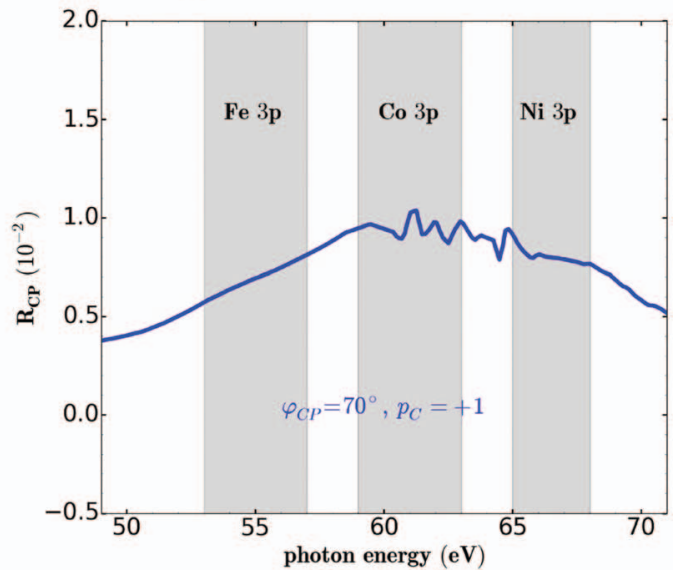

FIG. 3. (a) Simulated degree of circular polarization $p_{C}$ after the circular polarizer as a function of rotation angle $\varphi_{C P}$ for three photon energies corresponding to the $3 p$ absorption edges of $\mathrm{Fe}, \mathrm{Co}$, and $\mathrm{Ni}$. The circular polarizer was optimized for the Co $3 p$ absorption edge, thus showing the highest degree of circular polarization for $\varphi_{C P} \approx 70^{\circ}, 250^{\circ}\left(p_{C}=+1\right)$, and $\varphi_{C P} \approx 110^{\circ}, 290^{\circ}\left(p_{C}=-1\right)$. (b) Overall reflectivity $R_{C P}$ of the circular polarizer for $p_{C}=+1$ around the $3 p$ absorption edges of $\mathrm{Fe}, \mathrm{Co}$, and $\mathrm{Ni}$ with a strong magneto-optical signal ${ }^{6}$ (grey boxes).

\section{B. Design of the instrument for generation and analysis of polarized EUV light and circular magnetic dichroism measurements between $50 \mathrm{eV}$ and $70 \mathrm{eV}$ photon energy}

The entire optical setup is mounted on an optical breadboard inside a vacuum chamber. For the Bragg mirror linear polarizer, we used a commercial piezo-driven, vacuumcompatible holder for $1 \mathrm{in}$. mirrors (Smaract STT-25). During the alignment, the holder allows for tilting the mirror around the vertical and horizontal axes by $\pm 2.5^{\circ}$.

The subsequent optical element, the triple-reflection circular polarizer, required a specially designed holder for fixed mounting of three mirrors (two mirrors $10 \mathrm{~mm} \times 30 \mathrm{~mm}$, one mirror $10 \mathrm{~mm} \times 23 \mathrm{~mm}$ ) for $20^{\circ}-40^{\circ}-20^{\circ}$ grazing incidence. ${ }^{23}$ Thus, no further relative alignment of the mirrors is necessary. The holder consists of two parts secured together using screws (Fig. 4). Inside the cutouts of the two parts (two cutouts in one part, one cutout in the other part) the mirrors consisting of $20 \mathrm{~nm}$ Mo layer thermally evaporated on Si substrate are attached using glue. The entire device is mounted on a piezo-driven, vacuumcompatible rotational stage (Smaract SR-7012-S, minimum step size of $0.2 \times 10^{-3 \circ}$ ) with positioning control. For polarization analysis using a Rabinovitch polarimeter, ${ }^{24}$ we designed a special Bragg mirror holder for 1 in. optics allowing $42^{\circ}$ incidence angle with respect to the normal (Fig. 4) corresponding to the Brewster angle at $60.5 \mathrm{eV}$ (Co $3 p$ absorption edge). Similar to the triple-reflection circular polarizer, the holder is mounted on a piezo-driven, vacuum-compatible rotational stage (Smaract SR-5714-S, minimum step size of $0.16 \times 10^{-3 \circ}$ ) equipped with positioning sensors. We also designed a holder for the AXUV 100G photodiode $(10 \mathrm{~mm}$ $\times 10 \mathrm{~mm}$ active area) with mechanical support for the electrical SMA connector. The photodiode holder is attached to the analyzer holder and rotates with the analyzer around the beam axis.

All optical elements were prealigned using visible light. We performed the angular alignment of the Bragg mirror linear polarizer optimizing the reflectivity at $60.5 \mathrm{eV}$. For that purpose, we rotated the vacuum chamber around the central axis in the surface plane of the linear polarizer simultaneously recording EUV spectra. Once the intensity of emission lines

(a) Triple reflection circular polarizer

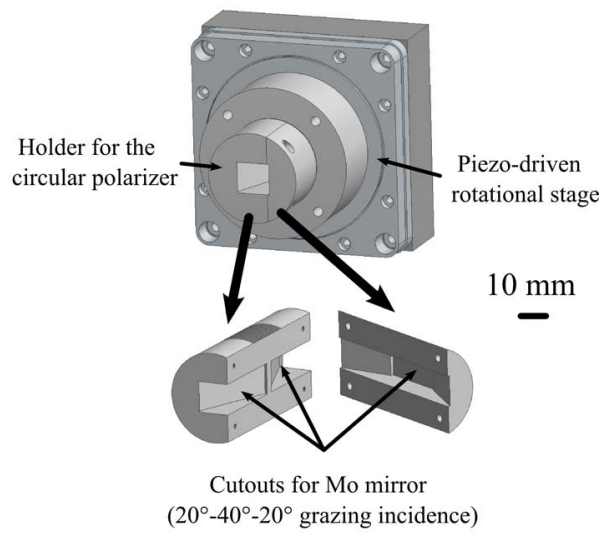

(b) Analyzer

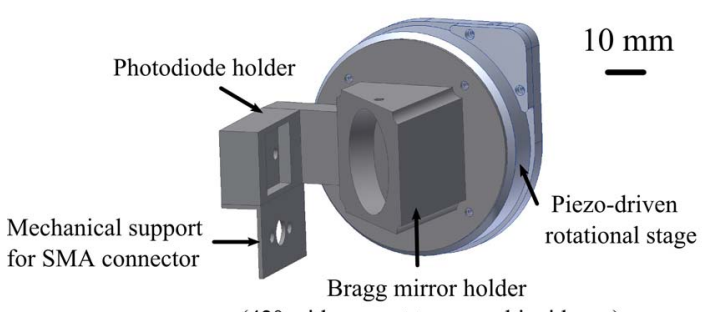

$\left(42^{\circ}\right.$ with respect to normal incidence)

FIG. 4. (a) Holder for the triple reflection circular polarizer consisting of two halves. All mirrors comprising a Mo $(20 \mathrm{~nm})$ layer on Si substrate are glued inside the milled parts. The whole device is attached to a piezo-driven rotational stage with positioning sensors. (b) Analyzer consisting of a Bragg mirror holder for $42^{\circ}$ with respect to the normal incidence (Brewster angle at $60.5 \mathrm{eV}$ ) and a photodiode holder. The whole device mounted on a piezodriven rotational stage with positioning control is rotated around the beam axis (Rabinovitch polarimeter ${ }^{24}$ ). 
at $60.5 \mathrm{eV}$ was highest, the alignment goal was reached. We note that the linear polarizer accepts a wide angle range between $\Theta=35^{\circ}$ and $\Theta=50^{\circ}$ without significant loss of the degree of linear polarization (Fig. 2(b), blue curve).

For X-ray magnetic circular dichroism measurements at the Co $3 p$ absorption edge, we removed the Bragg mirror analyzer and placed a $\mathrm{Co} / \mathrm{Pt}$ multilayer sample between the pole shoes of a ferromagnetic yoke to magnetize it. We designed the yoke with a bore hole of $2 \mathrm{~mm}$ diameter for transmission measurements and magnetized the soft ferromagnetic yoke by a coil wound around the yoke. When supplying 4 A current to the coil, the magnetic field reaches a maximum of $320 \mathrm{mT}$ between the pole shoes with a $2 \mathrm{~mm}$ gap.

For the Co/Pt multilayer sample $(10 \mathrm{~mm} \times 10 \mathrm{~mm})$ with a $\mathrm{Si}_{3} \mathrm{~N}_{4}$ window $(0.5 \mathrm{~mm} \times 0.5 \mathrm{~mm})$, we designed a separate holder for transmission experiments. To align the $\mathrm{Co} / \mathrm{Pt}$ multilayer sample between the pole shoes of the yoke, we placed the sample on a two-dimensional piezo-driven linear stage for vertical and horizontal movement.

\section{CHARACTERIZATION OF THE PLASMA EMISSION SPECTRUM BEHIND THE BRAGG MIRROR LINEAR POLARIZER}

For our measurements, we employed a gas-discharge plasma EUV light source developed at the Fraunhofer Institute for Laser Technology ${ }^{18-20}$ and specifically designed for water window operation $(280 \mathrm{eV}-530 \mathrm{eV})$. In our experiments, the source parameters were $3.5 \mathrm{kV}$ discharge voltage, $2 \mu \mathrm{F}$ total capacity and $20 \mathrm{~Hz}$ pulse repetition rate. In order to produce high intensity radiation between $50 \mathrm{eV}$ and $70 \mathrm{eV}$ photon energy, we used EUV radiation emitted from highly ionized nitrogen $\left(\mathrm{N}^{3+}, \mathrm{N}^{4+}\right)$ and oxygen atoms $\left(\mathrm{O}^{4+}, \mathrm{O}^{5+}\right)$. A typical spectrum measured by a grazing-incidence EUV spectrometer with a blazed, spherical, gold-coated grating (1200 lines/mm) and a back-illuminated CCD camera (Andor iKon-M) is presented in Fig. 5. The measured relative spectral bandwidth of single emission lines $\Delta \lambda / \lambda \approx 10^{-3}$ is limited by the spectral resolution of our spectrometer. We note that emis-

(a)

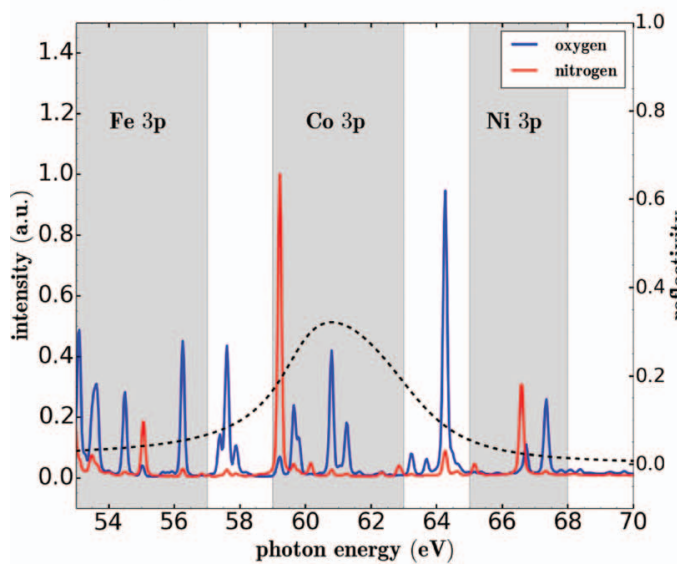

sion lines of multiply ionized nitrogen and oxygen atoms are well in the range of $3 p$ absorption edges of $\mathrm{Fe}, \mathrm{Co}$, and $\mathrm{Ni}$. Furthermore, based on reported synchrotron measurements, the magneto-optical resonances are known to occur in the vicinity of the absorption edges and to be spectrally broader than the absorption edge itself, having a spectral width of few $\mathrm{eV}$ for the $3 p$ absorption edges of $\mathrm{Fe}, \mathrm{Co}$, and $\mathrm{Ni}^{5,6}$ (grey marked regions in Fig. 5 extracted from Ref. 6). Therefore, it is possible to use nitrogen and oxygen plasma radiation for element-selective magneto-optical polarization spectroscopy and microscopy at the $3 p$ absorption edges of the $3 d$ ferromagnets. In the case of $\mathrm{Co}$, multiple emission lines of oxygen plasma are within the $3 p$ magneto-optical resonance used in our magnetic circular dichroism measurements.

We selected oxygen and nitrogen emission lines by a Bragg mirror linear polarizer with peak reflectivity at $60.5 \mathrm{eV}$ (Fig. 5(b)). The pulse energy of the oxygen and nitrogen emission lines around $60.5 \mathrm{eV}$ measured with a calibrated EUV photodiode after the Bragg mirror is about $1.3 \mathrm{~mJ} / \mathrm{sr}$ $\left(1.4 \times 10^{14}\right.$ photons $\left./ \mathrm{sr}\right)$ for the oxygen and $0.8 \mathrm{~mJ} / \mathrm{sr}(0.8$ $\times 10^{14}$ photons/sr) for the nitrogen plasma. To obtain the highest possible magnetic contrast in our XMCD studies, we used the oxygen instead of the nitrogen lines located very close to $60.5 \mathrm{eV}$ (grey box in Fig. 5(b)). The intense oxygen line at $64.3 \mathrm{eV}$ only contributes to the overall intensity but not to the magnetic signal.

\section{POLARIMETRIC MEASUREMENT OF THE DEGREE OF LINEAR AND CIRCULAR POLARIZATION AT $60 \mathrm{eV}$}

We measured the polarization properties of EUV light around the $3 p$ absorption edge of $\mathrm{Co}(60 \mathrm{eV})$ behind the linear and circular polarizer. Below we first describe the measurements of the degree of linear polarization and then that of circular polarization.

Similar to Eq. (2) in Sec. II A, we define the degree of linear polarization $p_{L}$ after the linear polarizer as

$$
p_{L}=\frac{S_{1, L}}{S_{0, L}},
$$

(b)

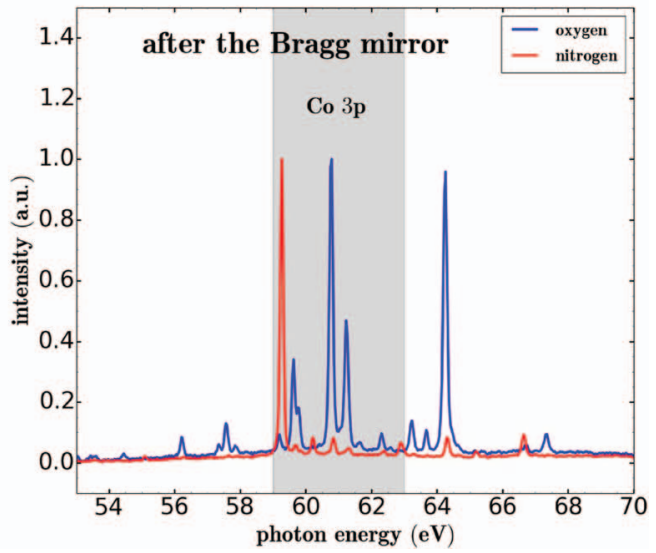

FIG. 5. (a) Spectra of highly ionized nitrogen $\left(\mathrm{N}^{3+}, \mathrm{N}^{4+}\right)$ and oxygen $\left(\mathrm{O}^{4+}, \mathrm{O}^{5+}\right)$ atoms between $53 \mathrm{eV}$ and $70 \mathrm{eV}$ as emitted from the gas-discharge plasmabased EUV light source. The grey boxes indicate the spectral region of magneto-optical resonances around the $3 p$ absorption edges of Fe, Co, and Ni. ${ }^{6}$ The dashed line corresponds to the reflectivity of the Bragg mirror linear polarizer for $60.5 \mathrm{eV}$ at $42^{\circ}$ normal incidence (Brewster angle). (b) Spectra of highly ionized nitrogen and oxygen atoms measured directly after the Bragg mirror linear polarizer for $60.5 \mathrm{eV}$ comprising $\left[\mathrm{Si}(9.09 \mathrm{~nm}) / \mathrm{B}_{4} \mathrm{C}(6.06 \mathrm{~nm})\right]_{50 \mathrm{x}}$. 
where $S_{0, L}$ and $S_{1, L}$ denote the first two values of the Stokes vector after the linear polarizer $\left(S_{2, L}=0\right)$. For completely spolarized light, $p_{L}=-1$. The photodiode signal $I_{L P}$ can be readily derived using the Stokes formalism and it reads

$$
I_{L P}\left(\varphi_{A}\right)=I_{L P, 0}\left[1-p_{L} \times \cos \left(2 \psi_{A}\right) \times \cos \left(\varphi_{A}\right)\right],
$$

where $I_{L P, 0}$ is a constant factor.

The influence of the analyzer is taken into account by

$$
\psi_{A}=\tan ^{-1}\left(\frac{\left|r_{p, A}\right|}{\left|r_{s, A}\right|}\right),
$$

with $r_{p, A}$ and $r_{s, A}$ being the complex reflectivities for $\mathrm{p}$ - and s-polarized light. For our analyzer placed at the Brewster angle, we assume $\left|r_{p, A}\right| /\left|r_{s, A}\right| \approx 0$. Therefore, according to Eq. (7), $\psi_{A} \approx 0$ and thus $\cos \left(2 \psi_{A}\right)=1$. We define the analyzer rotation angle $\varphi_{A}$ (Fig. 1) in such a way that for $\varphi_{A}=0$ the s-p-coordinate systems of the linear polarizer and analyzer are identical and therefore $I_{L P}\left(\varphi_{A}=0\right)$ is at maximum.

To measure the degree of linear polarization $p_{L}$ (Eq. (5)), we placed the Bragg mirror analyzer directly behind the Bragg mirror linear polarizer and then rotated the analyzer clockwise with respect to the beam propagation direction around the beam axis by an angle $\varphi_{A}$ (Fig. 6(a)). In order to suppress photon energies below $30 \mathrm{eV}$ being also reflected by the Bragg mirror, we inserted an $\mathrm{Al}(100 \mathrm{~nm}) /$ Parylene $\mathrm{N}$ $(100 \mathrm{~nm})$ spectral filter into the beam path. We detected the signal by an EUV photodiode (AXUV 100G) and amplified with a low-noise current amplifier (FEMTO DLPCA-200) by a factor of $10^{9}$ V/A. Finally, we measured the time-integrated voltage by a voltmeter (HP 3457A). For each angle position $\varphi_{A}$, we took an average of four measurements. In addition, we separately recorded the offset voltage for every angle position in order to correct our data for rotational stage positiondependent voltage variations. A representative measurement is shown in Fig. 6(a). We fitted multiple data sets according to Eq. (6) and obtained an average value of $p_{L}=-(0.94$ $\pm 0.04)$. Although the measured degree of linear polarization is slightly lower than predicted by simulations, it is sufficient for conversion of linearly to circularly polarized light.

To analyze the polarization properties of our triplereflection circular polarizer, we modeled the Müller matrix of the circular polarizer as

$$
M_{C P}=\left(\begin{array}{cccc}
a & b & 0 & 0 \\
b & a & 0 & 0 \\
0 & 0 & c \times \cos \left(\Delta_{C P}\right) & c \times \sin \left(\Delta_{C P}\right) \\
0 & 0 & -c \times \sin \left(\Delta_{C P}\right) & c \times \cos \left(\Delta_{C P}\right)
\end{array}\right) \text {. }
$$

The ansatz for the matrix $M_{C P}$ stems from the multiplication of three standard Müller matrixes for all three Mo mirrors. ${ }^{21}$ Here, the parameters $a, b$, and $c$ depend on ellipsometric parameters $\psi$ of the three Mo mirrors, whereas the parameter $c$ depends on $a$ and $b$, and can be readily determined from these parameters (see the Appendix). The phase shift $\Delta_{C P}$ denotes the total phase shift between the s- and pcomponent after all three reflections. The circular polarizer was designed for $\Delta_{C P}=-90.9^{\circ}$ at $60.5 \mathrm{eV}$ for efficient conversion of linearly to circularly polarized light (Sec. II A). The photodiode signal $I_{C P}\left(\varphi_{A}\right)$ behind the analyzer follows the equation

$$
\begin{aligned}
I_{C P}\left(\varphi_{A}\right)= & I_{C P, 0} \times\left[a-b \times \cos \left(2 \varphi_{C P}\right)-b \times \cos \left(2 \varphi_{A}+2 \varphi_{C P}\right)\right. \\
& \left.+0.5 \times a \times \cos \left(2 \varphi_{A}+4 \varphi_{C P}\right)+0.5 \times a \times \cos \left(2 \varphi_{A}\right)\right] .
\end{aligned}
$$

Similar to Eq. (6), $I_{C P, 0}$ is a constant factor, $\varphi_{A}$ the rotation angle of the analyzer (clockwise with respect to the beam direction), and $\varphi_{C P}$ the rotation angle of the circular polarizer (counterclockwise with respect to the beam direction). Equation (9) assumes a completely s-polarized light behind the linear polarizer, i.e., $S_{1, L}=-S_{0, L}$ and as before in Eq. (6), $\psi_{A}$ $\approx 0$. Furthermore, we fixed $\Delta_{C P}=-90.9^{\circ}$ and neglected small terms proportional to $c \cdot \cos \left(\Delta_{C P}\right)$ in Eq. (9). (a)

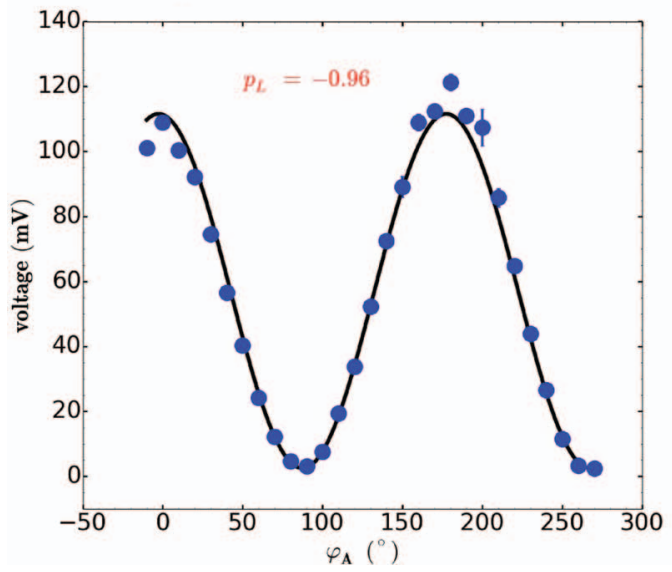

(b)

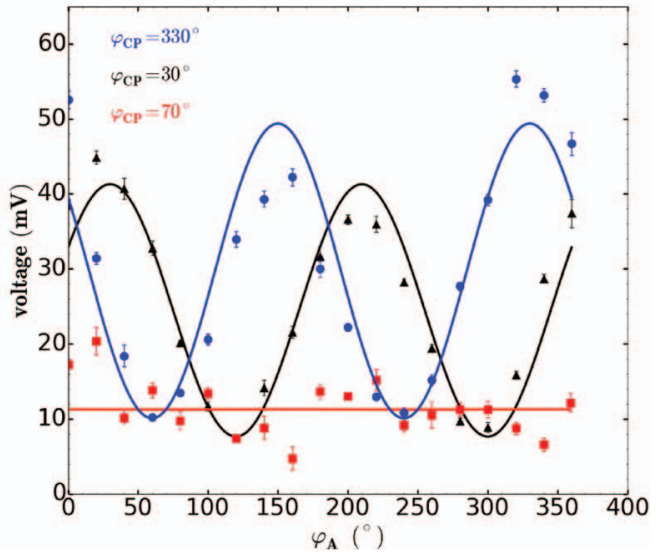

FIG. 6. (a) Measurement of the degree of linear polarization with emission lines from oxygen (Fig. 5(b)) around $60.5 \mathrm{eV}$ photon energy (blue dots) and fit (black line) according to Eq. (6). The signal of the photodiode is plotted versus the analyzer rotation angle $\varphi_{A}$. The degree of linear polarization extracted from the fit amounts to $p_{L}=-0.96$. (b) Measurement of the degree of circular polarization with emission lines from oxygen around $60.5 \mathrm{eV}$ behind the triple reflection circular polarizer. The photodiode voltage is shown as a function of the analyzer rotating angle $\varphi_{A}$ (dots) for different rotation angles $\varphi_{C P}$ of the circular polarizer. The lines are fits according to Eq. (9). For $\varphi_{C P}=70^{\circ}$ (red dots), the signal is independent of $\varphi_{A}$ confirming that the degree of circular polarization is at maximum. 
Fitting the measured intensity $I_{C P}\left(\varphi_{A}\right)$ from Eq. (9) for various fixed rotation angles $\varphi_{C P}$ (Fig. 6(b)), we extracted the parameters $a$ and $b$ and then calculated the dependent parameter $c$. The details of our analysis are summarized in the Appendix. In the next step, we calculated the degree of circular polarization according to

$$
p_{C}=\frac{c \times \sin \left(\Delta_{C P}\right) \times \sin \left(2 \varphi_{C P}\right)}{b \times \cos \left(2 \varphi_{C P}\right)-a} .
$$

To measure the Müller matrix parameters of our circular polarizer, we placed it between the linear polarizer and analyzer and independently rotated both circular polarizer and analyzer around the beam axis. We applied the same measurement technique as for the linear polarizer, i.e., the photodiode signal was amplified with a low-noise current amplifier by a factor of $10^{9} \mathrm{~V} / \mathrm{A}$ and the resulting voltage was measured by a time-integrating voltmeter. We fixed the rotation angle $\varphi_{C P}$ (counterclockwise with respect to the beam direction) and scanned $\varphi_{A}$ (clockwise with respect to the beam direction) between $0^{\circ}$ and $360^{\circ}$ in $20^{\circ}$ steps. For accurate polarization analysis, we separately measured the voltage offset for each $\varphi_{A}$ with the EUV light source being off. A representative result for $\varphi_{C P}=30^{\circ}, 70^{\circ}$, and $330^{\circ}$ is displayed in Fig. 6(b). The periodic voltage modulations present for $\varphi_{C P}=30^{\circ}$ and $\varphi_{C P}=330^{\circ}$ disappear when $\varphi_{C P}=70^{\circ}$, i.e., the photodiode signal does not depend on the analyzer angle $\varphi_{A}$. For this rotation angle, we efficiently convert linearly to circularly polarized light at $60.5 \mathrm{eV}$ as expected from simulations (Sec. II A). From measurements at six different angles $\varphi_{C P}$, we determined the parameters $a, b$, and $c$ and derived a maximum value of $p_{C}=0.81 \pm 0.15$ for $\varphi_{C P}=70^{\circ}$ and $\Delta_{C P}=-90.9^{\circ}$.

\section{XMCD MEASUREMENTS ON A Co/Pt-MULTILAYER FILM AT THE COBALT 3p ABSORPTION EDGE (60 eV)}

For the XMCD studies, we placed the coil and magnetic yoke with a bore hole for transmission measurements directly behind the Bragg mirror linear and circular polarizers and mounted the sample on two piezo-driven linear stages for vertical and horizontal movement in the center between the pole shoes of the yoke. As a suitable test sample for our XMCD measurements we chose $[\mathrm{Co}(0.8 \mathrm{~nm}) / \mathrm{Pt}(1.4 \mathrm{~nm})]_{16 \mathrm{x}}$ layers grown on $\mathrm{Si}_{3} \mathrm{~N}_{4}(50 \mathrm{~nm}) / \mathrm{Pt}(5 \mathrm{~nm})$ by ion beam sputtering ${ }^{25,26}$ and capped with $\mathrm{Pt}(0.6 \mathrm{~nm})$. A Co/Pt multilayer film exhibits a large perpendicular uniaxial anisotropy ${ }^{25,26}$ and therefore, can be magnetized out-of-plane, which, for normal incidence of light, ensures a strong XMCD signal at the Co $3 p$ absorption edge. From the scientific perspective, a $\mathrm{Co} / \mathrm{Pt}$ multilayer film is a highly interesting ferromagnetic layer system for several reasons. Most importantly, within a certain thickness range of $\mathrm{Co}$ and $\mathrm{Pt}$ layers, the magnetization oriented perpendicular or even canted with respect to the film plane tends to split into many alternatingly oriented ferromagnetic domains with the average domain size of about $100 \mathrm{~nm}$ at zero external magnetic field. ${ }^{25,26}$ The latter property renders Co/Pt multilayer films an ideal model system for studies of laser heating effects on the ferromagnetic domain structure employing high harmonics ${ }^{27}$ as well as for studies of femtosecond mag- netization dynamics of nanometer scale domains with a freeelectron laser. $^{28,29}$

The magnetization curve of the Co/Pt multilayer sample measured with polar magneto-optical Kerr effect (P-MOKE) is shown in the inset of Fig. 7. In order to saturate the magnetization, we applied $320 \mathrm{mT}$ magnetic field perpendicular to the sample surface. In our measurements, we first set the circular polarizer to $\varphi_{C P}=70^{\circ}\left(p_{C}=+1\right)$, alternately applied $\pm 320 \mathrm{mT}$ magnetic field, and then recorded the transmitted signal $I^{ \pm}( \pm 320 \mathrm{mT})$ on the CCD camera for both magnetic fields. We note that the actual image on the CCD camera is the beam profile after the $\mathrm{Co} / \mathrm{Pt}$ multilayer sample including all oxygen spectral lines reflected by the Bragg mirror linear polarizer (Fig. 5(b)). For further data analysis, the signal was binned along one spatial direction in the region of interest. The difference of the transmitted intensity averaged over 50 measurements for each magnetic field (10 s or 200 pulses per measurement), is shown in Fig. 7. For the backgroundcorrected XMCD asymmetry $A_{X M C D}$ calculated according to equation

$$
A_{X M C D}=\frac{I^{+}(+320 \mathrm{mT})-I^{-}(-320 \mathrm{mT})}{I^{+}(+320 \mathrm{mT})+I^{-}(-320 \mathrm{mT})},
$$

we obtained $A_{X M C D}=+(2.7 \pm 0.1) \%$.

After that, we changed the rotation angle of the circular polarizer to $\varphi_{C P}=110^{\circ}\left(p_{C}=-1\right)$ and repeated the above described measurement procedure. As expected for the XMCD effect, the difference signal (Fig. 7) and thus the asymmetry keeps the same magnitude but changes its sign. We measured $A_{X M C D}=-(2.8 \pm 0.1) \%$. To validate our data, we calculated the expected XMCD asymmetry from the magneto-optical absorption $\Delta \beta$ of the refractive index

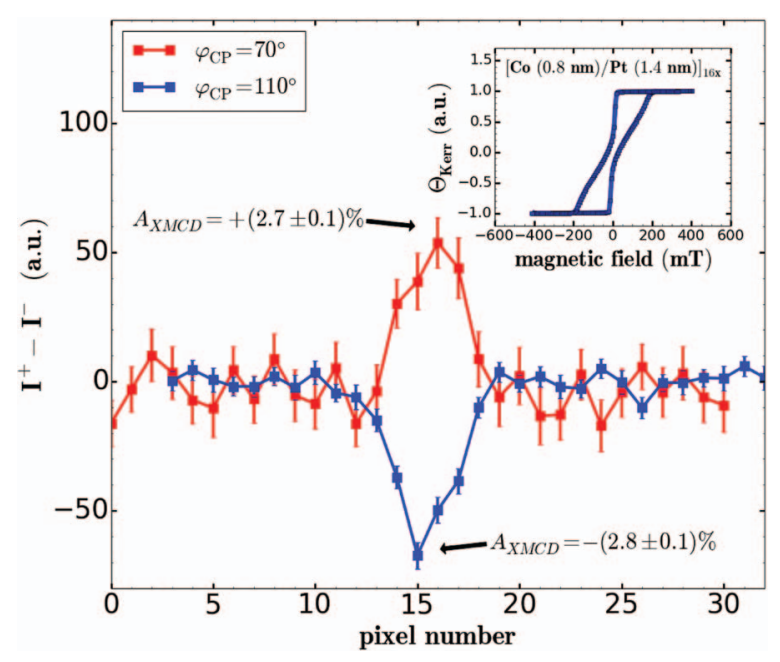

FIG. 7. XMCD difference signal $I^{+}(+320 \mathrm{mT})-I^{-}(-320 \mathrm{mT})$ as recorded by the CCD camera for $\varphi_{C P}=70^{\circ}\left(p_{C}=+1\right)$ and $\varphi_{C P}=110^{\circ}\left(p_{C}=\right.$ $-1)$. We note that the graph displays a beam profile including all oxygen spectral lines reflected by the Bragg mirror linear polarizer (Fig. 5(b)). For the background-corrected XMCD asymmetry, we obtained $A_{X M C D}$ $=+(2.7 \pm 0.1) \%$ and $A_{X M C D}=-(2.8 \pm 0.1) \%$ for different helicities. The inset shows the magnetization curve (Kerr rotation $\Theta_{\text {Kerr }}$ ) of a [Co $(0.8$ $\mathrm{nm}) / \mathrm{Pt}(1.4 \mathrm{~nm})]_{16 \mathrm{x}}$ multilayer measured by polar magneto-optical Kerr effect (P-MOKE) with visible light (350 nm wavelength). Using our magnetic yoke with a maximum field of $320 \mathrm{mT}$ the sample can be magnetized to saturation. 


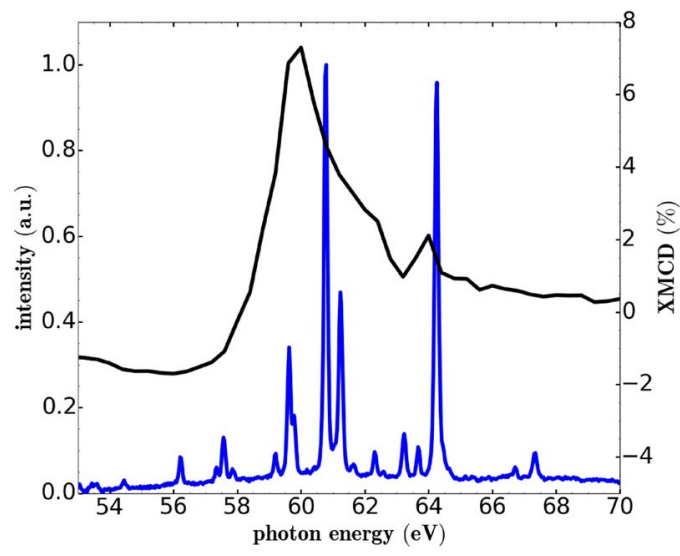

FIG. 8. XMCD spectrum for Co (total thickness $d=12.8 \mathrm{~nm}$ ) calculated by Eq. (12) from experimentally determined imaginary part of magnetic refractive index ${ }^{6}$ (black curve) and oxygen spectrum of the gas-discharge plasmabased EUV light source behind the Bragg mirror linear polarizer (blue curve)

$n=1-(\delta+\Delta \delta)+i \times(\beta+\Delta \beta)$ and a total Co thickness of $d=12.8 \mathrm{~nm}$ according to ${ }^{4}$

$$
A_{X M C D}=2 \frac{E}{\hbar c} d \times \Delta \beta
$$

where $E$ denotes the photon energy, $\hbar$ the reduced Planck constant, and $c$ the speed of light. Contrary to other magnetooptical effects like the MOKE or the Faraday effect, the XMCD asymmetry depends on one single magneto-optical parameter $\Delta \beta$. To simulate $A_{X M C D}$ in the vicinity of the Co $3 p$ absorption edge, we inserted the photon energy-dependent parameter $\Delta \beta$ recently measured by Valencia et $a l .{ }^{6}$ in Eq. (12). The expected XMCD asymmetry is plotted together with the oxygen spectrum behind the Bragg mirror linear polarizer in Fig. 8. The expected asymmetry of a few percent agrees well with our data. We note that our XMCD signal represents an average over several spectral lines of the oxygen plasma (Fig. 5(b)).

To further confirm our results, we studied the magnetic field dependence of the intensity difference signal $I^{+}\left(\mu_{0} H_{r e f}\right)$ $-I^{-}\left(\mu_{0} H\right)$ defining a fixed reference magnetic field $\mu_{0} H_{r e f}$ $=+320 \mathrm{mT}$ and six variable magnetic fields $\mu_{0} H(-320 \mathrm{mT}$, $-200 \mathrm{mT},-100 \mathrm{mT}, 0 \mathrm{mT}, 100 \mathrm{mT}$, and $200 \mathrm{mT}$ ). The intensity difference averaged over 10 measurements, each $10 \mathrm{~s}$ or 200 pulses, is displayed in Fig. 9.

As expected for $\mu_{0} H_{r e f}=+320 \mathrm{mT}$ from the magnetization curve (inset in Fig. 7), the difference signal is present for $\mu_{0} H<0$ and disappears for $\mu_{0} H>0$.

In summary, we generated circularly polarized light with both helicities from a laboratory-based plasma EUV light source and measured XMCD asymmetry values at the Co $3 p$ absorption edge comparable to the reported synchrotron studies. 5 The changing sign of the XMCD asymmetry upon helicity reversal confirms the magnetic origin of the signal. Moreover, we changed the magnetic field and observed a magnetic signal, which follows the magnetization curve. Our results, to our knowledge, are the first laboratory-based XMCD measurements at the Co $3 p$ absorption edge with a plasma-based EUV light source.

\section{CONCLUSION AND OUTLOOK}

We simulated, designed, and characterized an instrument for generation of circularly polarized EUV light at the $3 p$ absorption edges of $\mathrm{Fe}, \mathrm{Co}$, and $\mathrm{Ni}(50 \mathrm{eV}-70 \mathrm{eV})$ employing a compact gas-discharge plasma-based EUV light source. For the first time in a laboratory-based experiment with a plasma-based EUV light source, we successfully measured the XMCD effect at the Co $3 p$ absorption edge $(60.5 \mathrm{eV})$ that previously was only possible at synchrotrons, at freeelectron lasers and with laser-generated high harmonics due
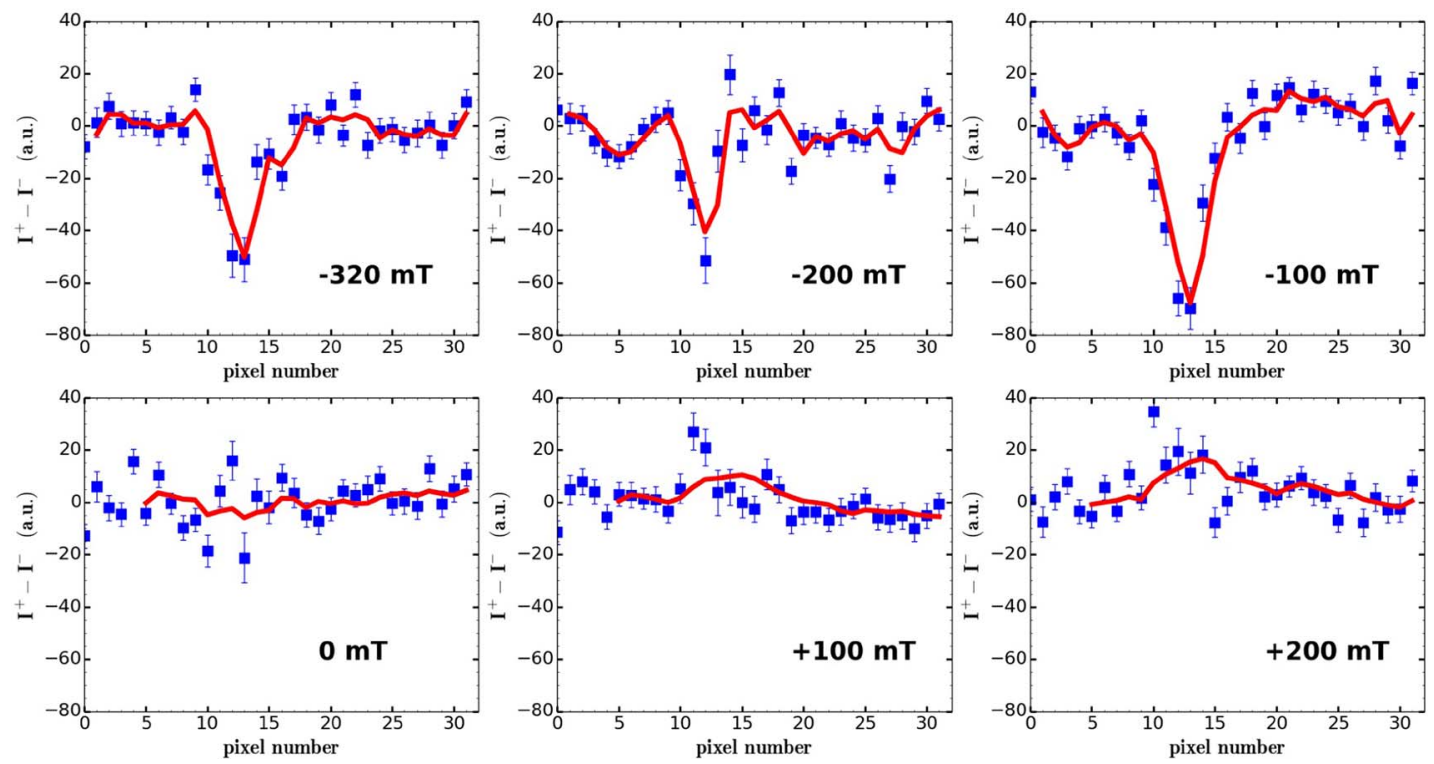

FIG. 9. Magnetic field dependence of the XMCD difference signal $I^{+}\left(\mu_{0} H_{r e f}\right)-I^{-}\left(\mu_{0} H\right)$ (blue: data points, red: smoothed curve) for $\mu_{0} H=-320 \mathrm{mT}$, $-200 \mathrm{mT},-100 \mathrm{mT}, 0 \mathrm{mT},+100 \mathrm{mT}$, and $+200 \mathrm{mT}$ for a fixed reference magnetic field $\mu_{0} H_{r e f}=+320 \mathrm{mT}$. Here, we fixed $\varphi_{C P}$ to $110^{\circ}$. The XMCD difference signal is only present for $\mu_{0} H<0$, i.e., for different signs of $\mu_{0} H_{\text {ref }}$ and $\mu_{0} H$ as expected from the magnetization curve (inset in Fig. 7). For $\mu_{0} H>0$, i.e., for the same sign of $\mu_{0} H_{r e f}$ and $\mu_{0} H$, no magnetic signal is expected according to the magnetization curve. 
to the lack of circularly polarized EUV radiation in the smallscale laboratory. Our results open the perspective for transfer of some synchrotron capabilities to the home laboratory using full time available high power plasma-based light sources. These light sources offer the advantages of photon energies in the EUV and soft X-ray spectral range at high intensity with multi-kHz repetition rate. The spectral position and intensity of the emission lines can be tuned by gas species and electrical discharge energy, which immediately allows elemental and chemical contrast at elemental absorption edges. In addition, our work demonstrates that the initially unpolarized light is easily converted to linearly and circularly polarized light required for magneto-optical polarization spectroscopy and microscopy. In future studies, it is straightforward to combine our polarization optics and a Fresnel zone plate EUV microscope..$^{30}$ In our concept, the polarization optics will be placed between the collector and the ferromagnetic sample, which domain structure will be imaged by the Fresnel zone plate. The proposed microscope will significantly advance the current imaging techniques and allow elementselective imaging of ferromagnetic domains at the $3 p$ absorption edges of $\mathrm{Fe}, \mathrm{Co}$, and $\mathrm{Ni}$ in the small-scale laboratory environment.

\section{ACKNOWLEDGMENTS}

L.J. acknowledges financial support by the Helmholtz Association for a Helmholtz Professorship as a part of the Pact for Research and Innovation. D.W., D.R., R.A., and L.J. also acknowledge financial support by JARA-FIT Seed Funds through the Excellence Initiative. Moreover, we thank Stefan Braun (Fraunhofer IWS Dresden) for Bragg mirror design and fabrication as well as Konstantin Tsigutkin for careful proofreading of the paper.

\section{APPENDIX: CALCULATION OF $p_{C}$}

The parameters $a$ and $b$ from Eq. (8) depend on the ellipsometric quantities $\psi$ (see Eq. (4)) of all three Mo mirrors of the circular polarizer in the following way:

$$
\begin{aligned}
& a=1+2 \times \cos \left(2 \psi_{40}\right) \times \cos \left(2 \psi_{20}\right)+\cos \left(2 \psi_{20}\right)^{2}, \\
& b=-2 \times \cos \left(2 \psi_{20}\right)-\cos \left(2 \psi_{40}\right)-\cos \left(2 \psi_{40}\right) \times \cos \left(2 \psi_{20}\right)^{2},
\end{aligned}
$$

where $\psi_{20}$ and $\psi_{40}$ denote the ellipsometric quantities for $20^{\circ}$ and $40^{\circ}$ grazing incidence, respectively. We solved this system of two nonlinear equations numerically for a fixed range of $\psi_{20}$ and $\psi_{40}$ and obtained $\psi_{20}$ and $\psi_{40}$ from measured parameters $a$ and $b$ (Sec. IV). Then, we calculated the parameter $c$ according to equation

$$
c=\sin \left(2 \psi_{20}\right)^{2} \times \sin \left(2 \psi_{40}\right)
$$

and, based on Eqs. (A1) and (A2) we derived the degree of circular polarization $p_{C}$ using Eq. (10).
${ }^{1}$ D. Attwood, Soft X-rays and Extreme Ultraviolet Radiation: Principles and Applications (Cambridge University Press, 1999).

${ }^{2}$ F. Scholze, C. Laubis, C. Buchholz, A. Fischer, S. Plöger, F. Scholz, and G. Ulm, Proc. SPIE 6151, 615137 (2006).

${ }^{3}$ S. Danylyuk, H.-S. Kim, P. Loosen, K. Bergmann, and L. Juschkin, J. Micro/Nanolith. MEMS MOEMS 12(3), 033002 (2013).

${ }^{4}$ H.-Ch. Mertins, S. Valencia, A. Gaupp, W. Gudat, P. M. Oppeneer, and C. M. Schneider, Appl. Phys. A 80, 1011 (2005).

${ }^{5}$ M. F. Tesch, M. C. Gilbert, H.-Ch. Mertins, D. E. Bürgler, U. Berges, and C. M. Schneider, Appl. Opt. 52(18), 4294 (2013).

${ }^{6}$ S. Valencia, A. Gaupp, W. Gudat, H.-C. Mertins, P. M. Oppeneer, D. Abramsohn, and C. M. Schneider, New J. Phys. 8, 254 (2006).

${ }^{7}$ L. Baumgarten, C. M. Schneider, H. Petersen, F. Schäfers, and J. Kirschner, Phys. Rev. Lett. 65, 492 (1990).

${ }^{8}$ F. U. Hillebrecht, Ch. Roth, H. B. Rose, M. Finazzi, and L. Braicovich, Phys. Rev. B 51, 9333 (1995).

${ }^{9}$ F. Schäfers, H.-Ch. Mertins, A. Gaupp, W. Gudat, M. Mertin, I. Packe, F. Schmolla, S. Di Fonzo, G. Soullié, W. Jark, R. Walker, X. Le Cann, R. Nyholm, and M. Eriksson, Appl. Opt. 38(19), 4074 (1999).

${ }^{10}$ T. Imazono, K. Sano, Y. Suzuki, T. Kawachi, and M. Koike, Rev. Sci. Instrum. 80(8), 085109 (2009).

${ }^{11}$ S. Kitamoto, H. Murakami, Y. Shishido, N. Gotoh, T. Shibata, K. Saito, T. Watanabe, J. Kanai, E. Takenaka, K. Nagasaki, M. Yoshida, D. Takei, and M. Morii, Rev. Sci. Instrum. 81, 023105 (2010).

${ }^{12}$ H. C. Kapteyn, M. M. Murnane, and I. P. Christov, Phys. Today 58(3), 39 (2005).

${ }^{13}$ W. B. Westerveld, K. Becker, P. W. Zetner, J. J. Corr, and J. W. McConkey, Appl. Opt. 24, 2256 (1985).

${ }^{14}$ B. Vodungbo, A. Barszczak Sardinha, J. Gautier, G. Lambert, C. Valentin, M. Lozano, G. Iaquaniello, F. Delmotte, S. Sebban, J. Lüning, and P. Zeitoun, Opt. Express 19(5), 4346 (2011)

${ }^{15}$ O. Kfir, P. Grychtol, E. Turgut, R. Knut, D. Zusin, D. Popmintchev, T. Popmintchev, H. Nembach, J. M. Shaw, A. Fleischer, H. Kapteyn, M. Murnane, and O. Cohen, e-print arXiv: 1401.4101.

${ }^{16} \mathrm{H}$. Höchst, R. Patel, and F. Middleton, Nucl. Instrum. Methods Phys. Res. A 347(1-3), 107 (1994).

${ }^{17}$ M. Suzuki, K. Hanmura, T. Kotani, N. Yamaguchi, M. Kobayashi, and A. Misu, Rev. Sci. Instrum. 66, 1589 (1995).

${ }^{18}$ K. Bergmann, G. Schriever, O. Rosier, M. Müller, W. Neff, and R. Lebert, Appl. Opt. 38, 5413 (1999).

${ }^{19}$ M. Benk and K. Bergmann, J. Micro/Nanolith. MEMS MOEMS 11(2), 021106 (2012).

${ }^{20}$ K. Bergmann, S. V. Danylyuk, and L. Juschkin, J. Appl. Phys. 106, 073309 (2009).

${ }^{21}$ H. Fujiwara, Spectroscopic Ellipsometry (Wiley, 2007).

${ }^{22}$ B. L. Henke, E. M. Gullikson, and J. C. Davis, At. Data Nucl. Data Tables 54(2), 181 (1993); see http://henke.lbl.gov/optical_constants/, from this database, we extracted the refractive indices of $\mathrm{Mo}, \mathrm{Si}, \mathrm{SiO}_{2}$, and $\mathrm{B}_{4} \mathrm{C}$ between $50 \mathrm{eV}$ and $70 \mathrm{eV}$ photon energy.

${ }^{23}$ V. G. Horton, E. T. Arakawa, R. N. Hamm, and M. W. Williams, Appl. Opt. 8(3), 667 (1969)

${ }^{24}$ K. Rabinovitch, L. R. Canfield, and R. P. Madden, Appl. Opt. 4(8), 1005 (1965).

${ }^{25}$ H. Stillrich, C. Menk, R. Frömter, and H. P. Oepen, J. Appl. Phys. 105, 07C308 (2009).

${ }^{26}$ D. Stickler, R. Frömter, H. Stillrich, C. Menk, H. P. Oepen, C. Gutt, S. Streit-Nierobisch, L.-M. Stadler, G. Grübel, C. Tieg, and F. Yakhou-Harris, Phys. Rev. B 84, 104412 (2011).

${ }^{27}$ C. Weier, R. Adam, D. Rudolf, R. Frömter, P. Grychtol, G. Winkler, A. Kobs, H. P. Oepen, H. C. Kapteyn, M. M. Murnane, and C. M. Schneider, "Femtosecond-laser-induced modifications in $\mathrm{Co} / \mathrm{Pt}$ multilayers studied with tabletop resonant magnetic scattering," Europhys. Lett. (submitted).

${ }^{28}$ B. Vodungbo, J. Gautier, G. Lambert, A. B. Sardinha, M. Lozano, S. Sebban, M. Ducousso, W. Boutu, K. Li, B. Tudu et al., Nat. Commun. 3, 999 (2012).

${ }^{29}$ S. Pfau, S. Schaffert, L. Müller, C. Gutt, A. Al-Shemmary, F. Büttner, R. Delaunay, S. Düsterer, S. Flewett, R. Frömter et al., Nat. Commun. 3, 1100 (2012)

${ }^{30}$ P. W. Wachulak, A. Bartnik, and H. Fiedorowicz, Opt. Lett. 35(14), 2337 (2010) 